\title{
Common Random Fixed Point theorem for compatible random multivalued operators
}

\author{
Dr. Neetu Vishwakarma
}

\begin{abstract}
The aim of this paper is to prove some common random fixed point theorem for two pairs of compatible random multivalued operators satisfying rational inequality.
\end{abstract}

Keywords: Random fixed point, Compatible maps, Polish space.

AMS Mathematics Subject Classification (2000): 47H10, 54H25.

\section{Introduction}

The systematic study of random equations employing the methods of functional analysis was first initiated by Prague School of Probabilistic in 1950's by Spacek [12] and Hans [7,8]. In separable metric space, random fixed point theorems for contraction mappings was proved by Spacek [12] and Hans [7,8]. BharuchaReid [6] generalized Mukherjee's [10] result on general probability measure space. For multivalued mappings Itoh [9] obtained random analogues of corresponding deterministic result for different classes of mappings. Papageoriou [11], Beg [2,3], Beg and Shahzad [5] and Beg and Abbas [4] proved some common random fixed point and random coincidence point of a pair of compatible random operators.

Preliminaries: Let $(\mathrm{X}, \mathrm{d})$ be a Polish space, that is a separable complete metric space and $(\Omega, a)$ be a measurable space. Let $2^{\mathrm{X}}$ be the family of all subsets of $\mathrm{X}$ and $\mathrm{CB}(\mathrm{X})$ denote the family of all nonempty bounded closed subsets of $\mathrm{X}$.

A mapping T: $\Omega \rightarrow 2^{\mathrm{X}}$, is called measurable, if for any open subset $\mathrm{C}$ of $\mathrm{X}$,

$$
\mathrm{T}^{-1}(\mathrm{C})=\{\omega \in \Omega: \mathrm{T}(\omega) \cap \mathrm{C} \neq \phi\} \in a .
$$

A mapping $\xi: \Omega \rightarrow \mathrm{X}$ is called measurable selector of a measurable mapping $\quad \mathrm{T}: \Omega \rightarrow 2^{\mathrm{X}}$, if $\xi$ is measurable and for any $\omega \in \Omega, \xi(\omega) \in \mathrm{T}(\omega)$. measurable.

A mapping $\mathrm{T}: \Omega \times \mathrm{X} \rightarrow \mathrm{CB}(\mathrm{X})$ is called random multivalued operator, if for every $\mathrm{x} \in \mathrm{X}, \mathrm{T}(., \mathrm{x})$ is

A mapping $\mathrm{f}: \Omega \times \mathrm{X} \rightarrow \mathrm{X}$ is called random operator, if for every $\mathrm{x} \in \mathrm{X}, \mathrm{f}(., \mathrm{x})$ is measurable.

A measurable mapping $\xi: \Omega \rightarrow \mathrm{X}$, is called the random fixed point of a random multivalued operator $\mathrm{T}: \Omega \times \mathrm{X} \rightarrow \mathrm{CB}(\mathrm{X})(\mathrm{f}: \Omega \times \mathrm{X} \rightarrow \mathrm{X}$ ), if for every $\omega \in \Omega, \quad \xi(\omega) \in \mathrm{T}(\omega, \xi(\omega))(\mathrm{f}(\omega, \xi(\omega))=\xi(\omega))$.

A measurable mapping $\xi: \Omega \rightarrow \mathrm{X}$ is a random coincident point of

$\mathrm{T}: \Omega \times \mathrm{X} \rightarrow \mathrm{CB}(\mathrm{X})$ and $\mathrm{f}: \Omega \times \mathrm{X} \rightarrow \mathrm{CB}(\mathrm{X})$ if for any $\omega \in \Omega$,

$$
f(\omega, \xi(\omega))=T(\omega, \xi(\omega)) \text {. }
$$

Mappings $f, g: X \rightarrow X$ are compatible if $\lim _{n \rightarrow \infty} d\left(f g\left(x_{n}\right), g f\left(x_{n}\right)\right)=0$, provided that $\lim _{n \rightarrow \infty} f\left(x_{n}\right)$ and $\lim _{n \rightarrow \infty} g\left(x_{n}\right)$ exists in $X$ and $\lim _{n \rightarrow \infty} f\left(x_{n}\right)=\lim _{n \rightarrow \infty} g\left(x_{n}\right)$.

Random operators $\mathrm{S}, \mathrm{T}: \Omega \times \mathrm{X} \rightarrow \mathrm{X}$ are compatible if $\mathrm{S}(\omega,$.$) and \mathrm{T}(\omega,$.$) are compatible for each$ $\omega \in \Omega$. (See Beg and Shahzad [5])

Main Result.

Theorem. Let $\mathrm{X}$ be a Polish space and let $(\mathrm{S}, \mathrm{T})$ and $(\mathrm{Q}, \mathrm{T})$ be two pairs of compatible random multivalued operators from $\Omega \times \mathrm{X} \rightarrow \mathrm{CB}(\mathrm{X})$ with $\mathrm{S}(\omega, \mathrm{X}) \subset \mathrm{T}(\omega, \mathrm{X})$ and $\mathrm{Q}(\omega, \mathrm{X}) \subset \mathrm{T}(\omega, \mathrm{X})$ for each $\omega \in \Omega$ and

$$
\begin{aligned}
H(S(\omega, x), Q(\omega, y)) \leq & \alpha(\omega) \frac{[d(T(\omega, x), S(\omega, x))]^{3}+[d(T(\omega, y), Q(\omega, y))]^{3}}{[d(T(\omega, x), S(\omega, x))]^{2}+[d(T(\omega, y), Q(\omega, y))]^{2}} \\
& +\beta(\omega) \frac{[d(T(\omega, x), S(\omega, x))]^{2}+[d(T(\omega, y), Q(\omega, y))]^{2}}{[d(T(\omega, x), S(\omega, x))]+[d(T(\omega, y), Q(\omega, y))]} \\
& +\gamma(\omega) d(T(\omega, x), T(\omega, y))
\end{aligned}
$$


for each $\mathrm{x}, \mathrm{y} \in \mathrm{X}$ and $\omega \in \Omega$ where $\alpha, \beta, \gamma: \Omega \rightarrow(0,1)$ are measurable mapping such that $\alpha(\omega)+\beta(\omega)+$ $\gamma(\omega)<1$.

If one of the random multivalued operators $\mathrm{S}, \mathrm{Q}$ and $\mathrm{T}$ is continuous, then $\mathrm{S}, \mathrm{Q}$ and $\mathrm{T}$ have unique common random fixed point. (Here $\mathrm{H}$ represents the Hausdorff metric on $\mathrm{CB}(\mathrm{X})$ induced by the metric $\mathrm{d}$ ).

Proof. Let $\xi_{0}: \Omega \rightarrow \mathrm{X}$ be an arbitrary measurable mapping and choose a measurable mapping $\xi_{1}$ : $\Omega \rightarrow$ X such that $\mathrm{S}\left(\omega, \xi_{0}(\omega)\right)=\mathrm{T}\left(\omega, \xi_{1}(\omega)\right)$ for each $\omega \in \Omega$.

It further implies that there exists a measurable mapping $\xi_{2}: \Omega \rightarrow \mathrm{X}$ such that for any $\omega \in \Omega$

$\mathrm{Q}\left(\omega, \xi_{1}(\omega)\right)=\mathrm{T}\left(\omega, \xi_{2}(\omega)\right)$.

In general, we can choose measurable mappings $\xi_{2 \mathrm{n}+1}$ and $\xi_{2 \mathrm{n}+2}$ from $\Omega \rightarrow \mathrm{X}$ such that

$\mathrm{S}\left(\omega, \xi_{2 \mathrm{n}}(\omega)\right)=\mathrm{T}\left(\omega, \xi_{2 \mathrm{n}+1}(\omega)\right)$

and

$$
\mathrm{Q}\left(\omega, \xi_{2 \mathrm{n}+1}(\omega)\right)=\mathrm{T}\left(\omega, \xi_{2 \mathrm{n}+2}(\omega)\right)
$$

for each $\omega \in \Omega$ and $n=0,1,2, \ldots$.

Then for each $\omega \in \Omega$,

$$
\mathrm{d}\left(\mathrm{T}\left(\omega, \xi_{2 \mathrm{n}+1}(\omega)\right), \mathrm{T}\left(\omega, \xi_{2 \mathrm{n}+2}(\omega)\right)=\mathrm{H}\left(\mathrm{S}\left(\omega, \xi_{2 \mathrm{n}}(\omega)\right), \mathrm{Q}\left(\omega, \xi_{2 \mathrm{n}+1}(\omega)\right)\right.\right.
$$

$$
\begin{aligned}
& \leq \alpha(\omega) \frac{\left[d\left(T\left(\omega, \xi_{2 n}(\omega)\right), S\left(\omega, \xi_{2 n}(\omega)\right)\right)\right]^{3}+\left[d\left(T\left(\omega, \xi_{2 n+1}(\omega)\right), Q\left(\omega, \xi_{2 n+1}(\omega)\right)\right)\right]^{3}}{\left[d\left(T\left(\omega, \xi_{2 n}(\omega)\right), S\left(\omega, \xi_{2 n}(\omega)\right)\right)\right]^{2}+\left[d\left(T\left(\omega, \xi_{2 n+1}(\omega)\right), Q\left(\omega, \xi_{2 n+1}(\omega)\right)\right)\right]^{2}} \\
&+ \beta(\omega) \frac{\left[d\left(T\left(\omega, \xi_{2 n}(\omega)\right), S\left(\omega, \xi_{2 n}(\omega)\right)\right)\right]^{2}+\left[d\left(T\left(\omega, \xi_{2 n+1}(\omega)\right), Q\left(\omega, \xi_{2 n+1}(\omega)\right)\right)\right]^{2}}{\left[d\left(T\left(\omega, \xi_{2 n}(\omega)\right), S\left(\omega, \xi_{2 n}(\omega)\right)\right)\right]+\left[d\left(T\left(\omega, \xi_{2 n+1}(\omega)\right), Q\left(\omega, \xi_{2 n+1}(\omega)\right)\right)\right]} \\
&+\gamma(\omega) d\left(T\left(\omega, \xi_{2 n}(\omega)\right), T\left(\omega, \xi_{2 n+1}(\omega)\right)\right)
\end{aligned}
$$

$$
\begin{aligned}
\leq \alpha(\omega) & \frac{\left[\mathrm{d}\left(\mathrm{T}\left(\omega, \xi_{2 \mathrm{n}}(\omega)\right), \mathrm{T}\left(\omega, \xi_{2 \mathrm{n}+1}(\omega)\right)\right)\right]^{3}+\left[\mathrm{d}\left(\mathrm{T}\left(\omega, \xi_{2 \mathrm{n}+1}(\omega)\right), \mathrm{T}\left(\omega, \xi_{2 \mathrm{n}+2}(\omega)\right)\right)\right]^{3}}{\left[\mathrm{~d}\left(\mathrm{~T}\left(\omega, \xi_{2 \mathrm{n}}(\omega)\right), \mathrm{T}\left(\omega, \xi_{2 \mathrm{n}+1}(\omega)\right)\right)\right]^{2}+\left[\mathrm{d}\left(\mathrm{T}\left(\omega, \xi_{2 \mathrm{n}+1}(\omega)\right), \mathrm{T}\left(\omega, \xi_{2 \mathrm{n}+2}(\omega)\right)\right)\right]^{2}} \\
& +\beta(\omega) \frac{\left[\mathrm{d}\left(\mathrm{T}\left(\omega, \xi_{2 \mathrm{n}}(\omega)\right), \mathrm{T}\left(\omega, \xi_{2 \mathrm{n}+1}(\omega)\right)\right)\right]^{2}+\left[\mathrm{d}\left(\mathrm{T}\left(\omega, \xi_{2 \mathrm{n}+1}(\omega)\right), \mathrm{T}\left(\omega, \xi_{2 \mathrm{n}+2}(\omega)\right)\right)\right]^{2}}{\left[\mathrm{~d}\left(\mathrm{~T}\left(\omega, \xi_{2 \mathrm{n}}(\omega)\right), \mathrm{T}\left(\omega, \xi_{2 \mathrm{n}+1}(\omega)\right)\right)\right]+\left[\mathrm{d}\left(\mathrm{T}\left(\omega, \xi_{2 \mathrm{n}+1}(\omega)\right), \mathrm{T}\left(\omega, \xi_{2 \mathrm{n}+2}(\omega)\right)\right)\right]} \\
& +\gamma(\omega) \mathrm{d}\left(\mathrm{T}\left(\omega, \xi_{2 \mathrm{n}}(\omega)\right), \mathrm{T}\left(\omega, \xi_{2 \mathrm{n}+1}(\omega)\right)\right)
\end{aligned}
$$

$$
\begin{aligned}
& \leq \alpha(\omega) \frac{\left[\mathrm{d}\left(\mathrm{T}\left(\omega, \xi_{2 \mathrm{n}}(\omega)\right), \mathrm{T}\left(\omega, \xi_{2 \mathrm{n}+1}(\omega)\right)\right)+\mathrm{d}\left(\mathrm{T}\left(\omega, \xi_{2 \mathrm{n}+1}(\omega)\right), \mathrm{T}\left(\omega, \xi_{2 \mathrm{n}+2}(\omega)\right)\right)\right]^{3}}{\left[\mathrm{~d}\left(\mathrm{~T}\left(\omega, \xi_{2 \mathrm{n}}(\omega)\right), \mathrm{T}\left(\omega, \xi_{2 \mathrm{n}+1}(\omega)\right)\right)+\mathrm{d}\left(\mathrm{T}\left(\omega, \xi_{2 \mathrm{n}+1}(\omega)\right), \mathrm{T}\left(\omega, \xi_{2 \mathrm{n}+2}(\omega)\right)\right)\right]^{2}} \\
&+ \beta(\omega) \frac{\left[\mathrm{d}\left(\mathrm{T}\left(\omega, \xi_{2 \mathrm{n}}(\omega)\right), \mathrm{T}\left(\omega, \xi_{2 \mathrm{n}+1}(\omega)\right)\right)+\mathrm{d}\left(\mathrm{T}\left(\omega, \xi_{2 \mathrm{n}+1}(\omega)\right), \mathrm{T}\left(\omega, \xi_{2 \mathrm{n}+2}(\omega)\right)\right)\right]^{2}}{\left[\mathrm{~d}\left(\mathrm{~T}\left(\omega, \xi_{2 \mathrm{n}}(\omega)\right), \mathrm{T}\left(\omega, \xi_{2 \mathrm{n}+1}(\omega)\right)\right)+\mathrm{d}\left(\mathrm{T}\left(\omega, \xi_{2 \mathrm{n}+1}(\omega)\right), \mathrm{T}\left(\omega, \xi_{2 \mathrm{n}+2}(\omega)\right)\right)\right]} \\
&+\gamma(\omega) \mathrm{d}\left(\mathrm{T}\left(\omega, \xi_{2 \mathrm{n}}(\omega)\right), \mathrm{T}\left(\omega, \xi_{2 \mathrm{n}+1}(\omega)\right)\right)
\end{aligned}
$$

$\leq \alpha(\omega)\left[\mathrm{d}\left(\mathrm{T}\left(\omega, \xi_{2 \mathrm{n}}(\omega)\right), \mathrm{T}\left(\omega, \xi_{2 \mathrm{n}+1}(\omega)\right)+\mathrm{d}\left(\mathrm{T}\left(\omega, \xi_{2 \mathrm{n}+1}(\omega)\right), \mathrm{T}\left(\omega, \xi_{2 \mathrm{n}+2}(\omega)\right)\right)\right]\right.$

$+\beta(\omega)\left[\mathrm{d}\left(\mathrm{T}\left(\omega, \xi_{2 \mathrm{n}}(\omega)\right), \mathrm{T}\left(\omega, \xi_{2 \mathrm{n}+1}(\omega)\right)\right)+\mathrm{d}\left(\mathrm{T}\left(\omega, \xi_{2 \mathrm{n}+1}(\omega)\right), \mathrm{T}\left(\omega, \xi_{2 \mathrm{n}+2}(\omega)\right)\right)\right]$

$+\gamma(\omega) \mathrm{d}\left(\mathrm{T}\left(\omega, \xi_{2 \mathrm{n}}(\omega)\right), \mathrm{T}\left(\omega, \xi_{2 \mathrm{n}+1}(\omega)\right)\right)$

$\leq(\alpha(\omega)+\beta(\omega)+\gamma(\omega)) d\left(\mathrm{~T}\left(\omega, \xi_{2 \mathrm{n}}(\omega)\right), \mathrm{T}\left(\omega, \xi_{2 \mathrm{n}+1}(\omega)\right)\right)$

$+(\alpha(\omega)+\beta(\omega)) d\left(T\left(\omega, \xi_{2 n+1}(\omega)\right), T\left(\omega, \xi_{2 n+2}(\omega)\right)\right)$

i.e. $\quad(1-\alpha(\omega)-\beta(\omega)) d\left(T\left(\omega, \xi_{2 n+1}(\omega)\right), T\left(\omega, \xi_{2 n+2}(\omega)\right)\right)$

$$
\leq(\alpha(\omega)+\beta(\omega)+\gamma(\omega)) d\left(\mathrm{~T}\left(\omega, \xi_{2 \mathrm{n}}(\omega)\right), \mathrm{T}\left(\omega, \xi_{2 \mathrm{n}+1}(\omega)\right)\right)
$$

$\mathrm{d}\left(\mathrm{T}\left(\omega, \xi_{2 \mathrm{n}+1}(\omega)\right), \mathrm{T}\left(\omega, \xi_{2 \mathrm{n}+2}(\omega)\right)\right) \leq \mathrm{kd}\left(\mathrm{T}\left(\omega, \xi_{2 \mathrm{n}}(\omega)\right), \mathrm{T}\left(\omega, \xi_{2 \mathrm{n}+1}(\omega)\right)\right)$

where 


$$
\mathrm{k}=\frac{\alpha(\omega)+\beta(\omega)+\gamma(\omega)}{1-\alpha(\omega)-\beta(\omega)}<1
$$

Similarly,

$\mathrm{d}\left(\mathrm{T}\left(\omega, \xi_{2 \mathrm{n}+2}(\omega)\right), \mathrm{T}\left(\omega, \xi_{2 \mathrm{n}+3}(\omega)\right)\right) \leq \mathrm{kd}\left(\mathrm{T}\left(\omega, \xi_{2 \mathrm{n}+1}(\omega)\right), \mathrm{T}\left(\omega, \xi_{2 \mathrm{n}+2}(\omega)\right)\right.$

In general,

$$
\leq \mathrm{k}^{2} \mathrm{~d}\left(\mathrm{~T}\left(\omega, \xi_{2 \mathrm{n}}(\omega)\right), \mathrm{T}\left(\omega, \xi_{2 \mathrm{n}+1}(\omega)\right)\right.
$$

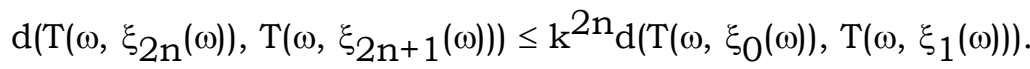

Furthermore $m>n$,

$\mathrm{d}\left(\mathrm{T}\left(\omega, \xi_{2 \mathrm{n}}(\omega)\right), \mathrm{T}\left(\omega, \xi_{2 \mathrm{~m}}(\omega)\right) \leq \mathrm{d}\left(\mathrm{T}\left(\omega, \xi_{2 \mathrm{n}}(\omega)\right), \mathrm{T}\left(\omega, \xi_{2 \mathrm{n}+1}(\omega)\right)\right)\right.$

$+\mathrm{d}\left(\mathrm{T}\left(\omega, \xi_{2 \mathrm{n}+1}(\omega)\right), \mathrm{T}\left(\omega, \xi_{2 \mathrm{n}+2}(\omega)\right)\right)+\ldots$

$+\mathrm{d}\left(\mathrm{T}\left(\omega, \xi_{2 \mathrm{~m}-1}(\omega)\right), \mathrm{T}\left(\omega, \xi_{2 \mathrm{~m}}(\omega)\right)\right)$

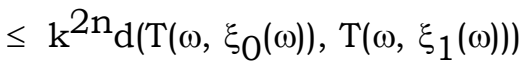

$+\mathrm{k}^{2 \mathrm{n}+1} \mathrm{~d}\left(\mathrm{~T}\left(\omega, \xi_{0}(\omega)\right), \mathrm{T}\left(\omega, \xi_{1}(\omega)\right)\right)+\ldots$

$+\mathrm{k}^{2 \mathrm{~m}-1} \mathrm{~d}\left(\mathrm{~T}\left(\omega, \xi_{0}(\omega)\right), \mathrm{T}\left(\omega, \xi_{1}(\omega)\right)\right)$

i.e. $d\left(T\left(\omega, \xi_{2 n}(\omega)\right), T\left(\omega, \xi_{2 m}(\omega)\right)\right) \leq \frac{k^{2 n}}{(1-\mathrm{k})} d\left(T\left(\omega, \xi_{0}(\omega)\right), T\left(\omega, \xi_{1}(\omega)\right)\right) \rightarrow 0 \quad$ as $n, m \rightarrow \infty$.

Thus $\left\{\mathrm{T}\left(\omega, \xi_{2 \mathrm{n}}(\omega)\right)\right\}$ and $\left\{\mathrm{T}\left(\omega, \xi_{2 \mathrm{~m}}(\omega)\right)\right\}$ are Cauchy sequence in $\mathrm{CB}(\mathrm{X})$, therefore there exists $\mathrm{A}(\omega)$ $\in \mathrm{CB}(\mathrm{X})$ such that

$\left\{\mathrm{T}\left(\omega, \xi_{2 \mathrm{n}}(\omega)\right)\right\} \rightarrow \mathrm{A}(\omega)$ for some $\omega \in \Omega$.

It further implies that $\left\{\mathrm{T}\left(\omega, \xi_{2 \mathrm{n}+1}(\omega)\right)\right\}$, \{S( $\left.\left(\omega, \xi_{2 \mathrm{n}}(\omega)\right)\right\}$ and $\left\{\mathrm{Q}\left(\omega, \xi_{2 \mathrm{n}+1}(\omega)\right)\right\}$ converges to $\mathrm{A}(\omega)$ for each $\omega \in \Omega$.

Let $\xi: \Omega \rightarrow$ X be a measurable mapping such that for each $\omega \in \Omega, \xi(\omega) \in \mathrm{A}(\omega)$.

Thus, we have

$$
\begin{aligned}
& \mathrm{T}\left(\omega, \xi_{2 \mathrm{n}+1}(\omega)\right) \rightarrow \mathrm{A}(\omega), \mathrm{S}\left(\omega, \xi_{2 \mathrm{n}}(\omega)\right) \rightarrow \mathrm{A}(\omega) \text { and } \\
& \mathrm{Q}\left(\omega, \xi_{2 \mathrm{n}+1}(\omega)\right) \rightarrow \mathrm{A}(\omega) \text { as } \mathrm{n} \rightarrow \infty .
\end{aligned}
$$

Now, suppose that $\mathrm{T}$ is continuous random multivalued operator, then

$\mathrm{T}\left(\omega, \mathrm{T}\left(\omega, \xi_{2 \mathrm{n}+1}(\omega)\right)\right) \rightarrow \mathrm{T}(\omega, \mathrm{A}(\omega)), \mathrm{T}\left(\omega, \mathrm{S}\left(\omega, \xi_{2 \mathrm{n}}(\omega)\right)\right) \rightarrow \mathrm{T}(\omega, \mathrm{A}(\omega))$ for every $\omega \in \Omega$.

Since pair $(\mathrm{S}, \mathrm{T})$ and $(\mathrm{Q}, \mathrm{T})$ are compatible random operator,

Since pair $(\mathrm{S}, \mathrm{T})$ and $(\mathrm{Q}, \mathrm{T})$ are compatible random operator, then for each $\omega \in \Omega$, we have

$\mathrm{T}\left(\omega, \mathrm{T}\left(\omega, \xi_{2 \mathrm{n}+1}(\omega)\right)\right) \rightarrow \mathrm{T}(\omega, \mathrm{A}(\omega)), \mathrm{S}\left(\omega, \mathrm{T}\left(\omega, \xi_{2 \mathrm{n}+1}(\omega)\right)\right) \rightarrow \mathrm{T}(\omega, \mathrm{A}(\omega))$

and $\mathrm{Q}\left(\omega, \mathrm{T}\left(\omega, \xi_{2 \mathrm{n}+1}(\omega)\right)\right) \rightarrow \mathrm{T}(\omega, \mathrm{A}(\omega))$.

Consider for each $\omega \in \Omega$

$\mathrm{H}\left(\mathrm{S}\left(\omega, \mathrm{T}\left(\omega, \xi_{2 \mathrm{n}}(\omega)\right)\right), \mathrm{Q}\left(\omega, \xi_{2 \mathrm{n}+1}(\omega)\right)\right)$

$\leq \alpha(\omega) \frac{\left[\mathrm{d}\left(\mathrm{T}\left(\omega, \mathrm{T}\left(\omega, \xi_{2 \mathrm{n}}(\omega)\right)\right), \mathrm{S}\left(\omega, \mathrm{T}\left(\omega, \xi_{2 \mathrm{n}}(\omega)\right)\right)\right]^{3}+\left[\mathrm{d}\left(\mathrm{T}\left(\omega, \xi_{2 \mathrm{n}+1}(\omega)\right)\right), \mathrm{Q}\left(\omega, \xi_{2 \mathrm{n}+1}(\omega)\right)\right)\right]^{3}}{\left[\mathrm{~d}\left(\mathrm{~T}\left(\omega, \mathrm{T}\left(\omega, \xi_{2 \mathrm{n}}(\omega)\right)\right), \mathrm{S}\left(\omega, \mathrm{T}\left(\omega, \xi_{2 \mathrm{n}}(\omega)\right)\right)\right]^{2}+\left[\mathrm{d}\left(\mathrm{T}\left(\omega, \xi_{2 \mathrm{n}+1}(\omega)\right)\right), \mathrm{Q}\left(\omega, \xi_{2 \mathrm{n}+1}(\omega)\right)\right)\right]^{2}}$

$+\beta(\omega) \frac{\left[\mathrm{d}\left(\mathrm{T}\left(\omega, \mathrm{T}\left(\omega, \xi_{2 \mathrm{n}}(\omega)\right)\right), \mathrm{S}\left(\omega, \mathrm{T}\left(\omega, \xi_{2 \mathrm{n}}(\omega)\right)\right)\right]^{2}+\left[\mathrm{d}\left(\mathrm{T}\left(\omega, \xi_{2 \mathrm{n}+1}(\omega)\right)\right), \mathrm{Q}\left(\omega, \xi_{2 \mathrm{n}+1}(\omega)\right)\right)\right]^{2}}{\left[\mathrm{~d}\left(\mathrm{~T}\left(\omega, \mathrm{T}\left(\omega, \xi_{2 \mathrm{n}}(\omega)\right)\right), \mathrm{S}\left(\omega, \mathrm{T}\left(\omega, \xi_{2 \mathrm{n}}(\omega)\right)\right)\right]+\left[\mathrm{d}\left(\mathrm{T}\left(\omega, \xi_{2 \mathrm{n}+1}(\omega)\right)\right), \mathrm{Q}\left(\omega, \xi_{2 \mathrm{n}+1}(\omega)\right)\right)\right]}$

$+\gamma(\omega) \mathrm{d}\left(\mathrm{T}\left(\omega, \mathrm{T}\left(\omega, \xi_{2 \mathrm{n}}(\omega)\right)\right), \mathrm{T}\left(\omega, \xi_{2 \mathrm{n}+1}(\omega)\right)\right)$.

On taking limit $\mathrm{n} \rightarrow \infty$ both sides, we get

$\mathrm{d}(\mathrm{T}(\omega, \mathrm{A}(\omega)), \mathrm{A}(\omega))$ 
$\leq \alpha(\omega) \frac{[\mathrm{d}(\mathrm{T}(\omega, \mathrm{A}(\omega)), \mathrm{T}(\omega, \mathrm{A}(\omega)))]^{3}+[\mathrm{d}(\mathrm{T}(\omega, \mathrm{A}(\omega)), \mathrm{T}(\omega, \mathrm{A}(\omega)))]^{3}}{[\mathrm{~d}(\mathrm{~T}(\omega, \mathrm{A}(\omega)), \mathrm{T}(\omega, \mathrm{A}(\omega)))]^{2}+[\mathrm{d}(\mathrm{T}(\omega, \mathrm{A}(\omega)), \mathrm{T}(\omega, \mathrm{A}(\omega)))]^{2}}$

$+\beta(\omega) \frac{[\mathrm{d}(\mathrm{T}(\omega, \mathrm{A}(\omega)), \mathrm{T}(\omega, \mathrm{A}(\omega)))]^{2}+[\mathrm{d}(\mathrm{T}(\omega, \mathrm{A}(\omega)), \mathrm{T}(\omega, \mathrm{A}(\omega)))]^{2}}{[\mathrm{~d}(\mathrm{~T}(\omega, \mathrm{A}(\omega)), \mathrm{T}(\omega, \mathrm{A}(\omega)))]+[\mathrm{d}(\mathrm{T}(\omega, \mathrm{A}(\omega)), \mathrm{T}(\omega, \mathrm{A}(\omega)))]}$

$+\gamma(\omega) \mathrm{d}(\mathrm{T}(\omega, \mathrm{A}(\omega)), \mathrm{A}(\omega))$

$(1-\gamma(\omega)) d(T(\omega, A(\omega)), A(\omega)) \leq 0$

$\mathrm{d}(\mathrm{T}(\omega, \mathrm{A}(\omega)), \mathrm{A}(\omega))=0$

i.e.

$\mathrm{T}(\omega, \mathrm{A}(\omega))=\mathrm{A}(\omega)$ for each $\omega \in \Omega$

But $\xi(\omega) \in \mathrm{A}(\omega)$.

Thus $\xi(\omega) \in \mathrm{T}(\omega, \xi(\omega))$.

Now, for any $\omega \in \Omega$

$\mathrm{H}\left(\mathrm{S}(\omega, \mathrm{A}(\omega)), \mathrm{Q}\left(\omega, \xi_{2 \mathrm{n}+1}(\omega)\right)\right)$

$\leq \alpha(\omega) \frac{[\mathrm{d}(\mathrm{T}(\omega, \mathrm{A}(\omega)), \mathrm{S}(\omega, \mathrm{A}(\omega)))]^{3}+\left[\mathrm{d}\left(\mathrm{T}\left(\omega, \xi_{2 \mathrm{n}+1}(\omega)\right), \mathrm{Q}\left(\omega, \xi_{2 \mathrm{n}+1}(\omega)\right)\right)\right]^{3}}{[\mathrm{~d}(\mathrm{~T}(\omega, \mathrm{A}(\omega)), \mathrm{S}(\omega, \mathrm{A}(\omega)))]^{2}+\left[\mathrm{d}\left(\mathrm{T}\left(\omega, \xi_{2 \mathrm{n}+1}(\omega)\right), \mathrm{Q}\left(\omega, \xi_{2 \mathrm{n}+1}(\omega)\right)\right)\right]^{2}}$

$+\beta(\omega) \frac{[\mathrm{d}(\mathrm{T}(\omega, \mathrm{A}(\omega)), \mathrm{S}(\omega, \mathrm{A}(\omega)))]^{2}+\left[\mathrm{d}\left(\mathrm{T}\left(\omega, \xi_{2 \mathrm{n}+1}(\omega)\right), \mathrm{Q}\left(\omega, \xi_{2 \mathrm{n}+1}(\omega)\right)\right)\right]^{2}}{[\mathrm{~d}(\mathrm{~T}(\omega, \mathrm{A}(\omega)), \mathrm{S}(\omega, \mathrm{A}(\omega)))]+\left[\mathrm{d}\left(\mathrm{T}\left(\omega, \xi_{2 \mathrm{n}+1}(\omega)\right), \mathrm{Q}\left(\omega, \xi_{2 \mathrm{n}+1}(\omega)\right)\right)\right]}$

$+\gamma(\omega) \mathrm{d}\left(\mathrm{T}(\omega, \mathrm{A}(\omega)), \mathrm{T}\left(\omega, \xi_{2 \mathrm{n}+1}(\omega)\right)\right)$.

Taking limit $\rightarrow \infty$, we get

$\mathrm{d}(\mathrm{S}(\omega, \mathrm{A}(\omega)), \mathrm{A}(\omega))$

$$
\begin{aligned}
& \leq \alpha(\omega) \frac{[\mathrm{d}(\mathrm{A}(\omega), \mathrm{S}(\omega, \mathrm{A}(\omega)))]^{3}+[\mathrm{d}(\mathrm{A}(\omega), \mathrm{A}(\omega))]^{3}}{[\mathrm{~d}(\mathrm{~A}(\omega), \mathrm{S}(\omega, \mathrm{A}(\omega)))]^{2}+[\mathrm{d}(\mathrm{A}(\omega), \mathrm{A}(\omega))]^{2}} \\
& +\beta(\omega) \frac{[\mathrm{d}(\mathrm{A}(\omega), \mathrm{S}(\omega, \mathrm{A}(\omega)))]^{2}+[\mathrm{d}(\mathrm{A}(\omega), \mathrm{A}(\omega))]^{2}}{[\mathrm{~d}(\mathrm{~A}(\omega), \mathrm{S}(\omega, \mathrm{A}(\omega)))]+[\mathrm{d}(\mathrm{A}(\omega), \mathrm{A}(\omega))]}
\end{aligned}
$$

$+\gamma(\omega) \mathrm{d}(\mathrm{A}(\omega), \mathrm{A}(\omega))$

$\mathrm{d}(\mathrm{S}(\omega, \mathrm{A}(\omega)), \mathrm{A}(\omega)) \leq \alpha(\omega) \mathrm{d}(\mathrm{A}(\omega), \mathrm{S}(\omega, \mathrm{A}(\omega)))+\beta(\omega) \mathrm{d}(\mathrm{A}(\omega), \mathrm{S}(\omega, \mathrm{A}(\omega)))$

$(1-\alpha(\omega)-\beta(\omega)) d(S(\omega, A(\omega)), A(\omega)) \leq 0$

i.e.

$\mathrm{d}(\mathrm{S}(\omega, \mathrm{A}(\omega)), \mathrm{A}(\omega)) \leq 0$

$\mathrm{S}(\omega, \mathrm{A}(\omega))=\mathrm{A}(\omega)$ for each $\omega \in \Omega$.

But $\xi(\omega) \in \mathrm{A}(\omega)$.

Thus, $\xi(\omega) \in \mathrm{S}(\omega, \mathrm{A}(\omega))$.

Finally,

$\mathrm{H}(\mathrm{S}(\omega, \mathrm{T}(\omega, \mathrm{A}(\omega)), \mathrm{Q}(\omega, \mathrm{A}(\omega)))$

$$
\begin{aligned}
& \leq \alpha(\omega) \frac{[\mathrm{d}(\mathrm{T}(\omega, \mathrm{A}(\omega)), \mathrm{S}(\omega, \mathrm{A}(\omega)))]^{3}+[\mathrm{d}(\mathrm{T}(\omega, \mathrm{A}(\omega)), \mathrm{Q}(\omega, \mathrm{A}(\omega)))]^{3}}{[\mathrm{~d}(\mathrm{~T}(\omega, \mathrm{A}(\omega)), \mathrm{S}(\omega, \mathrm{A}(\omega)))]^{2}+[\mathrm{d}(\mathrm{T}(\omega, \mathrm{A}(\omega)), \mathrm{Q}(\omega, \mathrm{A}(\omega)))]^{2}} \\
& +\beta(\omega) \frac{[\mathrm{d}(\mathrm{T}(\omega, \mathrm{A}(\omega)), \mathrm{S}(\omega, \mathrm{A}(\omega)))]^{2}+[\mathrm{d}(\mathrm{T}(\omega, \mathrm{A}(\omega)), \mathrm{Q}(\omega, \mathrm{A}(\omega)))]^{2}}{[\mathrm{~d}(\mathrm{~T}(\omega, \mathrm{A}(\omega)), \mathrm{S}(\omega, \mathrm{A}(\omega)))]+[\mathrm{d}(\mathrm{T}(\omega, \mathrm{A}(\omega)), \mathrm{Q}(\omega, \mathrm{A}(\omega)))]} \\
& +\gamma(\omega) \mathrm{d}(\mathrm{T}(\omega, \mathrm{A}(\omega)), \mathrm{T}(\omega, \mathrm{A}(\omega)))
\end{aligned}
$$

$\mathrm{d}(\mathrm{A}(\omega), \mathrm{Q}(\omega, \mathrm{A}(\omega)))$

$$
\leq \alpha(\omega) \frac{[\mathrm{d}(\mathrm{A}(\omega), \mathrm{A}(\omega))]^{3}+[\mathrm{d}(\mathrm{A}(\omega), \mathrm{Q}(\omega, \mathrm{A}(\omega)))]^{3}}{[\mathrm{~d}(\mathrm{~A}(\omega), \mathrm{A}(\omega))]^{2}+[\mathrm{d}(\mathrm{A}(\omega), \mathrm{Q}(\omega, \mathrm{A}(\omega)))]^{2}}
$$




$$
\begin{aligned}
& +\beta(\omega) \frac{[\mathrm{d}(\mathrm{A}(\omega), \mathrm{A}(\omega))]^{2}+[\mathrm{d}(\mathrm{A}(\omega), \mathrm{Q}(\omega, \mathrm{A}(\omega)))]^{2}}{[\mathrm{~d}(\mathrm{~A}(\omega), \mathrm{A}(\omega))]+[\mathrm{d}(\mathrm{A}(\omega), \mathrm{Q}(\omega, \mathrm{A}(\omega)))]} \\
& +\gamma(\omega) \mathrm{d}(\mathrm{A}(\omega), \mathrm{A}(\omega))
\end{aligned}
$$

$\mathrm{d}(\mathrm{A}(\omega), \mathrm{Q}(\omega, \mathrm{A}(\omega))) \leq \alpha(\omega) \mathrm{d}(\mathrm{A}(\omega), \mathrm{Q}(\omega, \mathrm{A}(\omega)))+\beta(\omega) \mathrm{d}(\mathrm{A}(\omega), \mathrm{Q}(\omega, \mathrm{A}(\omega)))$

$(1-\alpha(\omega)-\beta(\omega)) d(A(\omega), Q(\omega, A(\omega))) \leq 0$

i.e.

$\mathrm{d}(\mathrm{A}(\omega), \mathrm{Q}(\omega, \mathrm{A}(\omega))) \leq 0$

$\mathrm{Q}(\omega, \mathrm{A}(\omega))=\mathrm{A}(\omega)$ for each $\omega \in \Omega$.

But $\xi(\omega) \in \mathrm{A}(\omega)$.

Thus, $\xi(\omega) \in \mathrm{Q}(\omega, \mathrm{A}(\omega))$.

Hence, $\xi(\omega)$ is a random fixed point of random multivalued operator S, Q and T.

Uniqueness :

To prove uniqueness of common random fixed point of random multivalued operator.

Let $\xi_{1}, \xi_{2}: \Omega \rightarrow \mathrm{X}$ be two common random fixed point of random multivalued operators $\mathrm{S}$, Q and $\mathrm{T}$ such that $\xi_{1}(\omega)=\xi_{2}(\omega)$ for each $\omega \in \Omega$.

Consider for each $\omega \in \Omega$

$\mathrm{d}\left(\xi_{1}(\omega), \xi_{2}(\omega)\right) \leq \mathrm{H}\left(\mathrm{S}\left(\omega, \xi_{1}(\omega)\right), \mathrm{Q}\left(\omega, \xi_{2}(\omega)\right)\right)$

$$
\begin{gathered}
\leq \alpha(\omega) \frac{\left[\mathrm{d}\left(\mathrm{T}\left(\omega, \xi_{1}(\omega)\right), \mathrm{S}\left(\omega, \xi_{1}(\omega)\right)\right)\right]^{3}+\left[\mathrm{d}\left(\mathrm{T}\left(\omega, \xi_{2}(\omega)\right), \mathrm{Q}\left(\omega, \xi_{2}(\omega)\right)\right)\right]^{3}}{\left[\mathrm{~d}\left(\mathrm{~T}\left(\omega, \xi_{1}(\omega)\right), \mathrm{S}\left(\omega, \xi_{1}(\omega)\right)\right)\right]^{2}+\left[\mathrm{d}\left(\mathrm{T}\left(\omega, \xi_{2}(\omega)\right), \mathrm{Q}\left(\omega, \xi_{2}(\omega)\right)\right)\right]^{2}} \\
+\beta(\omega) \frac{\left[\mathrm{d}\left(\mathrm{T}\left(\omega, \xi_{1}(\omega)\right), \mathrm{S}\left(\omega, \xi_{1}(\omega)\right)\right)\right]^{2}+\left[\mathrm{d}\left(\mathrm{T}\left(\omega, \xi_{2}(\omega)\right), \mathrm{Q}\left(\omega, \xi_{2}(\omega)\right)\right)\right]^{2}}{\left[\mathrm{~d}\left(\mathrm{~T}\left(\omega, \xi_{1}(\omega)\right), \mathrm{S}\left(\omega, \xi_{1}(\omega)\right)\right)\right]+\left[\mathrm{d}\left(\mathrm{T}\left(\omega, \xi_{2}(\omega)\right), \mathrm{Q}\left(\omega, \xi_{2}(\omega)\right)\right)\right]} \\
+\gamma(\omega) \mathrm{d}\left(\mathrm{T}\left(\omega, \xi_{1}(\omega)\right), \mathrm{T}\left(\omega, \xi_{2}(\omega)\right)\right) \\
\mathrm{d}\left(\xi_{1}(\omega), \xi_{2}(\omega)\right) \leq \gamma(\omega) \mathrm{d}\left(\mathrm{T}\left(\omega, \xi_{1}(\omega)\right), \mathrm{T}\left(\omega, \xi_{2}(\omega)\right)\right) \\
(1-\gamma(\omega)) \mathrm{d}\left(\xi_{1}(\omega), \xi_{2}(\omega)\right) \leq 0 \\
\mathrm{~d}\left(\xi_{1}(\omega), \xi_{2}(\omega)\right) \leq 0 .
\end{gathered}
$$

Thus, $\xi_{1}(\omega)=\xi_{2}(\omega)$ for each $\omega \in \Omega$

which is a contradiction, so the result follows.

Corollary. Let $\mathrm{X}$ be a Polish space and let (S, P) and (T, Q) be two pairs of compatible random multivalued operators from $\Omega \times \mathrm{X} \rightarrow \mathrm{CB}(\mathrm{X})$ with $\mathrm{S}(\omega, \mathrm{X}) \subset \mathrm{Q}(\omega, \mathrm{X})$ and $\mathrm{T}(\omega, \mathrm{X}) \subset \mathrm{P}(\omega, \mathrm{X})$ for each $\omega \in \Omega$ and

$\omega \in \Omega$ and

$\mathrm{H}(\mathrm{S}(\omega, \mathrm{x}), \mathrm{T}(\omega, \mathrm{y}))$

$$
\begin{aligned}
& \leq \alpha(\omega) \frac{[\mathrm{d}(\mathrm{P}(\omega, \mathrm{x}), \mathrm{S}(\omega, \mathrm{x}))]^{3}+[\mathrm{d}(\mathrm{Q}(\omega, \mathrm{y}), \mathrm{T}(\omega, \mathrm{y}))]^{3}}{[\mathrm{~d}(\mathrm{P}(\omega, \mathrm{x}), \mathrm{S}(\omega, \mathrm{x}))]^{2}+[\mathrm{d}(\mathrm{Q}(\omega, \mathrm{y}), \mathrm{T}(\omega, \mathrm{y}))]^{2}} \\
& +\beta(\omega) \frac{[\mathrm{d}(\mathrm{P}(\omega, \mathrm{x}), \mathrm{S}(\omega, \mathrm{x}))]^{2}+[\mathrm{d}(\mathrm{Q}(\omega, \mathrm{y}), \mathrm{T}(\omega, \mathrm{y}))]^{2}}{[\mathrm{~d}(\mathrm{P}(\omega, \mathrm{x}), \mathrm{S}(\omega, \mathrm{x}))]+[\mathrm{d}(\mathrm{Q}(\omega, \mathrm{y}), \mathrm{T}(\omega, \mathrm{y}))]} \\
& +\gamma(\omega) \mathrm{d}(\mathrm{P}(\omega, \mathrm{x}), \mathrm{Q}(\omega, \mathrm{y}))
\end{aligned}
$$

for each $\mathrm{x}, \mathrm{y} \in \mathrm{X}$ and $\omega \in \Omega$, where $\alpha, \beta, \gamma: \Omega \rightarrow(0,1)$ are measurable mappings such that $\alpha(\omega)+\beta(\omega)+$ $\gamma(\omega)<1$. If one of the random multivalued operators $\mathrm{P}, \mathrm{Q}, \mathrm{T}$ or $\mathrm{S}$ is continuous then $\mathrm{P}, \mathrm{Q}, \mathrm{S}$ and $\mathrm{T}$ have unique common random fixed point (where $\mathrm{H}$ represents Hausdorff metric on $\mathrm{CB}(\mathrm{X})$ induced by metric $\mathrm{d}$ ).

\section{References}

[1.] Badshah, V.H. and Sayyed, Farkhunda, Common random fixed point of random multivalued operator on Polish spaces, Indian J. Pure App. Math. 33(4), Apr. 2002, 573-582.

[2.] Beg, I. Random fixed point of random operators satisfying semi-contractivity conditions, Mathematica Japonica 46(1997), no. 1, 151-155.

[3.] Beg, I., Approximation of random fixed point in normed spaces, Nonlinear Analysis, 51 (2002), No. 8, 1363-1372. 
[4.] Beg, I. and Abbas, Mujahid, Common random fixed point of compatible random operator, Int. J. Math. and Math. Sci. Vol. 2006 Article I.D. 23486, 1-15.

[5.] Beg, I, and Shahzad, N., Random fixed points of random multivalued operators on Polish spaces, Non-linear Analysis Theory Methods and Applications 20 (1993), 835-847.

[6.] Bharucha Reid, A.T.

[7.] Random integral equations, Academic Press, New York, 1972

[8.] Hans, O., Reduzierende zufallige transformation. Czechoslovak Mathematics Journal 7 (1957), 154-158.

[9.] Hans, O., Random operator equations, Proceeding of the $4^{\text {th }}$ Berkeley Symposium in Mathematical Statistics and Probability, Vol. II, Part I, University of California Press, California (1961), 185-202.

[10.] Itoh, S., A random fixed point theorem for a multivalued contraction mapping, Pacific Journal Mathematics 68 (1977), 85-90.

[11.] Mukherjee, A., Random transformations of Banach spaces. Ph.D. Dissertation, Wayne State University Detroit, Michegall, USA (1968.).

[12.] Papageorgiou, N.S., Random fixed point theorems for measurable multifunctions in Banach spaces, Proceedings of the American Mathematical Society 97 (1986), No. 3, 507-514.

[13.] Spacek, A., Zufallige Gleichungen, Czechoslovak Mathematical Journal 5 (1995), 462-466. 